\title{
Aircraft engine dust ingestion following sand storms
}

DOI:

10.1016/j.ast.2020.106072

\section{Document Version}

Accepted author manuscript

Link to publication record in Manchester Research Explorer

\section{Citation for published version (APA):}

Bojdo, N., Filippone, A., Parkes, B., \& Clarkson, R. (2020). Aircraft engine dust ingestion following sand storms. Aerospace Science and Technology, 106, 106072. https://doi.org/10.1016/j.ast.2020.106072

\section{Published in:}

Aerospace Science and Technology

\section{Citing this paper}

Please note that where the full-text provided on Manchester Research Explorer is the Author Accepted Manuscript or Proof version this may differ from the final Published version. If citing, it is advised that you check and use the publisher's definitive version.

\section{General rights}

Copyright and moral rights for the publications made accessible in the Research Explorer are retained by the authors and/or other copyright owners and it is a condition of accessing publications that users recognise and abide by the legal requirements associated with these rights.

\section{Takedown policy}

If you believe that this document breaches copyright please refer to the University of Manchester's Takedown Procedures [http://man.ac.uk/04Y6Bo] or contact uml.scholarlycommunications@manchester.ac.uk providing relevant details, so we can investigate your claim.

\section{OPEN ACCESS}




\title{
Aircraft engine dust ingestion following sand storms
}

\author{
Nicholas Bojdo, Antonio Filippone, Ben Parkes \\ The University of Manchester \\ Manchester M13 9PL \\ Rory Clarkson \\ Rolls-Royce CLE \\ Derby DE24 8BJ
}

\begin{abstract}
Commercial aircraft operating in some regions of the world are subject to harsh atmospheric conditions that can affect the efficiency and integrity of modern aero-engines. Conditions that are investigated in this contribution are dusty atmospheres following sand storms. Specifically, the dust ingested by the engines of an Airbus A380-841 (Rolls-Royce Trent 900) operating out of Doha airport (IATA: HIA) is estimated by simulating climb-out trajectories over three separate calendar months during which a number of sand storms have been identified. The atmosphere model incorporates dust concentration hind-casts from the European Centre for Medium-Range Weather Forecasts (ECMWF) near real-time Copernicus Atmosphere Monitoring Service database. A total of 365 flights were considered. The average dust mass ingested into the engine core per flight is estimated as $\sim 8.5 \mathrm{~g}$. Using a compressor fouling prediction model, the dust ingestion reduces the asymptotic deterioration rate time constant by a factor $\sim 8$. The worst dust storm within the time frame considered is selected for further examination. This flight is flagged as a dust-flight, during which the total mass of dust ingested into the engine core exceeds the monthly mean by two standard deviations. A peak dust ingestion rate of $22 \mathrm{mg} / \mathrm{s}$ occurs shortly after take-off, midway through the first climb segment. A second peak presents as the aircraft transitions into the climb from 3,000 feet to 10,000 feet. Finally, the dust ingested during a 20-minute holding pattern over the Persian Gulf is estimated as $\sim 8 \mathrm{~g}$, which is approximately the same as the dust ingested during climb-out on a very dusty day. The results suggest that a mission-based approach may be more useful for determining aircraft engine durability in dusty environments.
\end{abstract}

${ }^{*}$ Dept. Mechanical, Aerospace, Civil Engineering. Corresponding author: nicholas.bojdo@manchester.ac.uk 


\section{Nomenclature}

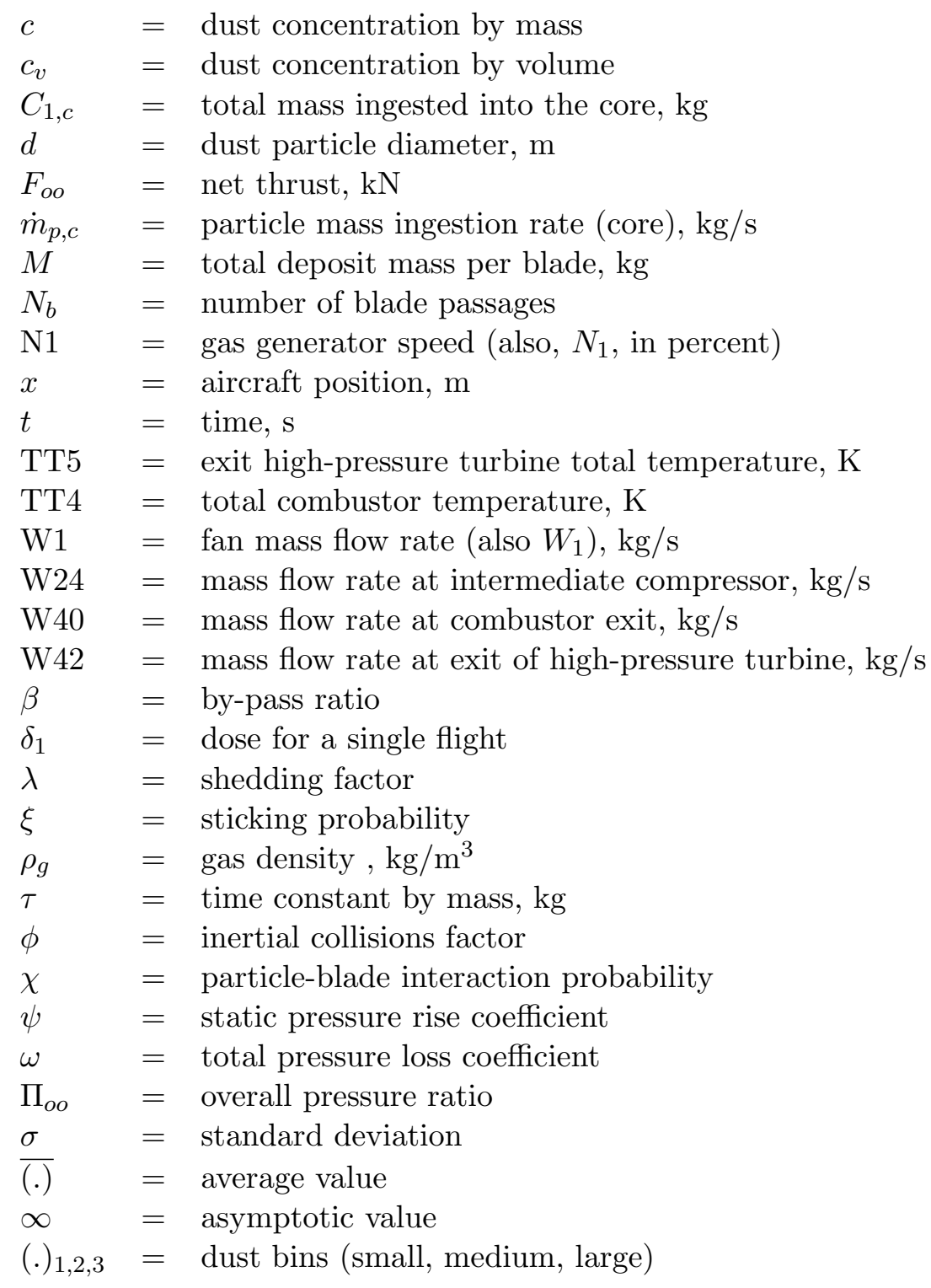

\section{Introduction}

The number of 100+ passenger-seat fleet in-service aircraft flying in and out of the Middle East is predicted to more than double over the next 20 years $^{1}$. The airports in this region are often characterised as non-benign due to the residual level of dust in the ambient air. The damage caused by regular movements in and out of these environments leads to a reduction in normal engine service intervals, and an increase in operating costs. The extent of the damage is a function of the dust mass loading and composition, as well as the engine's operating state.

Dust storms are defined as events in which visibility is reduced to $1 \mathrm{~km}$ or less as a result of blowing dust ${ }^{2}$. They can be created by advancing gust fronts or simply by strong winds that exceed the threshold velocity required to lift and transport significant quantities of particles. As a 
gust front, these storms appear like a giant advancing wall of sand reaching $1 \mathrm{~km}$ in height. When combined with weather system instabilities, the dust can be lofted as high as 20,000 feet into the atmosphere.

The Arabian Peninsula represents the second most productive source of atmospheric dust, contributing an estimated $11.8 \%$ of the total global dust burden according to a numerical study by Tanaka \& Chiba $^{3}$. A large study of 31 weather stations across the Arabian peninsula by Notaro et al. ${ }^{4}$ examined the historical dust activity in the region by recording dust-days over the period 1998-2013. A dust-day occurs if a dust or sand storm is observed at least once, or at least $25 \%$ of the daily observations indicate suspended dust. Dust activity appeared to follow a pronounced inter-annual to decadal variability, with a generally positive change in the annual frequency of dust-days since the turn of the $21^{\text {st }}$ century ${ }^{4}$.

One reason for the increase is the persistent drought occurring in the Fertile Crescent of Iraq and Syria; by comparing the occurrence of each dust-day for clusters of weather stations, including one at Doha International Airport, Notaro et al. were able to establish a clear trajectory of dust emanating from South-Eastern Iraq and washing over the west coast of the Persian Gulf ${ }^{4}$. This is significant, because the chemical composition of mineral dusts is dependent on the underlying geology of their origin. For example, squadrons in different parts of Saudi Arabia operating the helicopters with identical gas turbine engines with similar maintenance procedures have reported different levels and types of degradation ${ }^{5}$. Therefore one may not simply rely upon an in-situ ground sample as a means to anticipate the additional maintenance burden flying to and from a non-benign airport.

Aircraft climbing out of Middle Eastern airports will encounter a range of dust levels, owing to a variation in the frequency of dust storms throughout the year. In a study of 10 years' worth of meteorological data over Kuwait, Al-Dousari report a variation in the average number of dust storms per month across the years 2000-2010, from a low of around 0.3 in November to a peak of 4.5 storms in July ${ }^{6}$. Dust storms appear most frequent in the months of April to July. In some cases the dust cloud may be severe enough to ground aircraft, although not for reasons of engine damage: it is standard practice to close an airport if the visibility drops below $500 \mathrm{~m}$ because of the safety implications of flight crews not being able to see clearly other aircraft or obstructions. This corresponds to a dust concentration of $\sim 6 \mathrm{mg} / \mathrm{m}^{3}$, according to a simple correlation proposed by Shao et al. ${ }^{7}$, although $2 \mathrm{mg} / \mathrm{m}^{3}$ is a more reliable estimate.

This number is significant, because it was also used by aviation authorities to re-open airspace over Europe in 2010, that had been closed due to the emergence of a plume of volcanic ash. It was thought that concentration levels above $2 \mathrm{mg} / \mathrm{m}^{3}$ may have implications on safety margin, although estimates were based on very limited data. Since then, a more pragmatic approach to determine engine susceptibility to particle ingestion has been developed. Clarkson et al. ${ }^{8 ; 9}$ proposed a new approach, which considers the dose of a dust ingestion event instead, defined as the duration of 
exposure multiplied by the concentration of the particulate. This led to the adoption of a safe-to-fly dose limit of $14.4 \mathrm{gs} / \mathrm{m}^{3}$ being established, rather than a concentration limit of $2 \mathrm{mg} / \mathrm{m}^{3}$.

In developing the dose approach, Clarkson et al. compared several dozen in-service engine encounters with dust or volcanic ash, and used the severity of the resulting damage outcomes to establish 'long-term damage' and 'safe-to-fly' dose limits ${ }^{9}$. Doses for each encounter were calculated using re-analysis of dust concentration along the historical aircraft flight trajectory, and the severity of recorded damage was classified as negligible, long-term, exigent or safety implication. The authors generally found a non-linear increase in damage severity level with increasing dose, which allowed them to define dose boundaries between each category of damage severity.

Despite the similarity in concentration levels, there is no such requirement to certify the safe operation engines operating in dusty environments. This is most likely due to the difference in composition between volcanic ash and airborne mineral dust, which determines the adhesion characteristics and therefore the likelihood for blocking critical flow paths and creating a potential safety issue. However, over a period of time, an aircraft making frequent movements in and out of a non-benign airport will be subject to multiple doses of mineral dust that deposit on core gas path surfaces causing damage such as thermal-barrier coating spallation and sulphidation corrosion.

Adopting a dose-based approach to predict the lifetime of these components has not yet been explored in the literature, perhaps for good reason, but some authors have used it to rapidly assess the risk posed by plumes of volcanic ash to aviation. Prata et al. ${ }^{10}$ used a dispersion ensemble model to predict the ash concentration along an aircraft flight trajectory, with an associated level of uncertainty, and integrated over time spent in that concentration to calculate the total dose. The time step was determined from the aircraft velocity and distance travelled in each grid cell. The authors were able to estimate the probability and severity of the impact of the encounter on the aircraft, relative to the dose-based damage severity scale established by Clarkson \& Simpson ${ }^{9}$. To develop this scale, the Clarkson \& Simpson compared several dozen in-service engine encounters with dust or volcanic ash, and used the severity of the resulting damage outcomes to establish 'long-term damage' and 'safe-to-fly' dose limits ${ }^{9}$. Doses for each encounter were calculated using re-analysis of dust concentration along the historical aircraft flight trajectory, and correlated well with the resulting damage.

A limitation of the dose approach is that it does not differentiate between engine types (e.g. turbofan, turboshaft), engine operating point (e.g. take-off, climb) or dust type (e.g. volcanic ash, mineral dust). Each of these variables may affect the level of damage caused by a given dose of dust or ash. For example, many studies show that the likelihood of particle adhesion increases with engine core gas temperature, as the particle either melts, softens, or deforms plastically on impact more readily ${ }^{11 ; 12}$. Given that the design driver of minimum fuel burn pushes engines to higher total pressure-ratios and turbine inlet temperatures, cooling systems to ever-more intricate designs, and thermal barrier coatings to more locations, it is likely that a dose experienced by an engine 
of the 1980's damages a modern-day engine in very different ways. It would be difficult therefore to use the approach of Clarkson \& Simpson ${ }^{9}$ and Prata et a ${ }^{10}$ to predict engine susceptibility to low concentrations of mineral dust.

It is worth clarifying here the definition of the words dose and dosage, as both words have been used in the literature on this subject. Both words relate to the same thing - the product of concentration and duration - and are measured in units of gram-seconds per cubic metre (discussed also in Ref. ${ }^{10}$ ). In the field of medicine, dose is a specified amount taken at one time, while dosage is a prescribed amount, number, and frequency over a specified time period. For this reason, neither term is accurate. A more appropriate name would be the normalised dose, or the mass of dust ingested normalised by the unit of volume flow in which it was carried. Indeed, such a metric is very useful: it allows one to plot engines of different size on the same graph to quickly benchmark an exposure event against historical data, as has been successfully demonstrated by Clarkson \& Simpson ${ }^{9}$. It is perhaps less intuitive to use, though, when thinking about deposition rates and other phenomenological effects within the engine.

In the current work, we test the applicability of the dose approach to determine the durability of aircraft engines to mineral dust ingestion in arid environments. We propose a methodology to predict the particle mass ingestion rate for any given aircraft, operating a route in any given environment. We couple a flight performance code with a well-known openly-available atmospheric monitoring system to predict the dust ingested by an aircraft engine during climb-out. We apply the methodology to a commercial aircraft operating from an airport in the Middle East frequented by dust storms.

\section{Material and Methods}

\subsection{Flight Performance Model}

The trajectories, along with the remaining parts of the simulation, are calculated using the FLIGHT computer code, described in our earlier work ${ }^{13 ; 14 ; 15}$. It is recalled in this instance that flight trajectories are calculated iteratively on the basis of required range, payload, atmospheric conditions and other external factors. In the converged solution a near-optimal climb-out trajectory to the initial cruise altitude is generated following a straight path (in absence of navigation constraints), although in practice departures and arrivals can be optimised for minimum environmental effect ${ }^{16}$. In fact, the aircraft is flown over a great-circle trajectory using the conventional climb schedule. Turns and headings are of limited consequence, since the dust data are resolved on a grid no smaller than $40 \mathrm{~km} \times 40 \mathrm{~km}$ with a 6 -hour interval. Calculation of hold trajectories is done by selecting entry point (GPS coordinates, heading), flight altitude and duration. The remaining flight parameters are calculated with optimal flight procedures that minimise fuel burn.

The code is capable of predicting all major engine emissions; therefore, it is helpful in establishing the environmental effects of the aircraft. For the results presented in this paper, the 
code has been upgraded to deal with dust ingestion, with atmospheric conditions at departure taken from the Copernicus Atmosphere Monitoring Service (CAMS). However, no changes in aerothermodynamics are included as a result of the dust ingestion, since this is likely to be a long term effect rather than a catastrophic event occurring within 30 minutes of flight. The effects shown are for single flights rather than cumulative.

\subsection{Engine Model}

In lieu of a real Trent 900 engine model, we use a representative engine which is an early developmental engine and not similar to today's engine. Proprietary data have been provided by RollsRoyce on this engine, and used to validate the aero-thermodynamic performance model we have. This is necessary in order to establish the correct mass flows and temperatures. The mass flow rates are required to calculate the total dust ingestion and the temperatures are required to estimate the potential damage in critical sections of the engine. A one-dimensional aero-thermodynamic flow model is used. This variant of the Trent 900 has a by-pass ratio of 7.5 , an overall pressure ratio $\Pi_{o o}=38.97$ and a thrust rating $F_{o o}=334.70 \mathrm{kN}$. The fan design speed is $2,900 \mathrm{rpm}$; the certification documents state that the maximum fan speed at take-off is $97.2 \%$ of this value. Key data for this engine are available in the certification documents ${ }^{1}$.

Figure 1 shows the temperature TT4 at the exit of the combustor, prior to entry into the first stage of the high pressure turbine. A gap of $\sim 60 \mathrm{~K}$ is estimated between the simulation and the reference data at high engine rpm at this hot section of the engine. The difference in TT5 temperature, downstream of the low-pressure turbine, is just $\sim 20 \mathrm{~K}$, as illustrated in Figure 2 . The difference is limited to $\sim 20 \mathrm{~K}$ at high rpm.

In Figure 3 we show the total mass flow rate at the face of the engine, W1. The dashed line is a linear fit of the experimental data points (any other polynomial yields unrealistic correlations). At around 2,400 rpm, the mass flow data appear to flatten out, whilst the simulation led to a linear decrease in mass flow.

In Figure 4 selected mass flow rates are shown. As measured, these are W2.4 (mass flow rate at the inlet of the medium pressure compressor), W4.0 (mass flow rate at the exit of the combustor), W4.2 (mass flow rate at the section between the high-pressure turbine and the intermediate pressure turbine). The simulated data are W4.0. All lines are linear regressions of the data points. From this result, we established that the simulated engine has a core mass flow rate comparable with the real engine.

\footnotetext{
${ }^{1}$ EASA Type Certificate TCDS E.012, February 2019.
} 


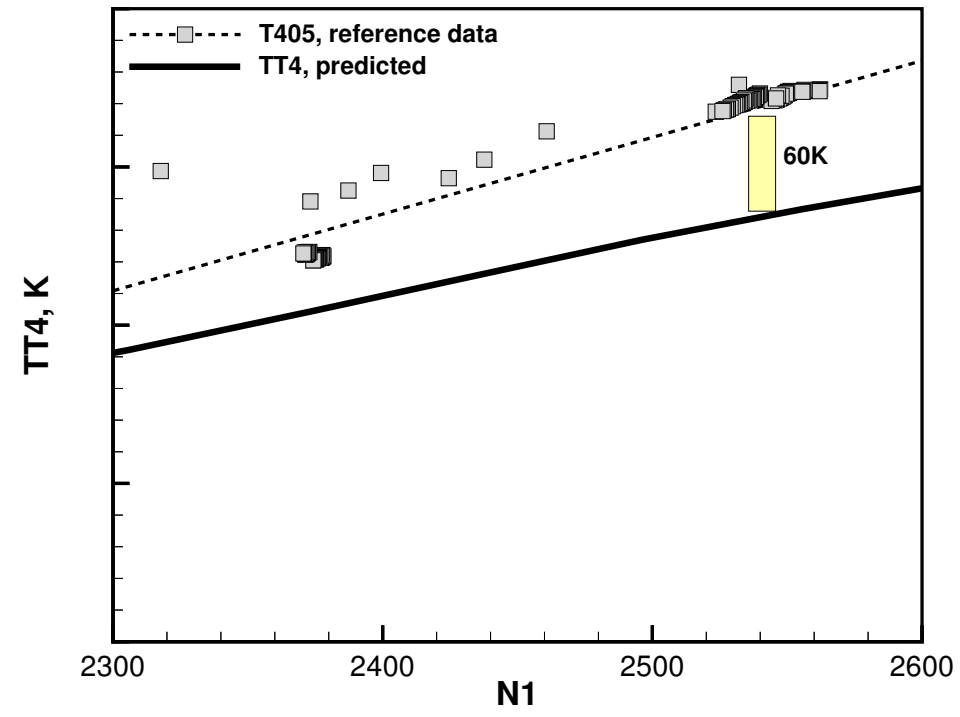

Figure 1: Engine Trent 900, simulated TT4 temperature and comparison with reference data from Rolls-Royce tests.

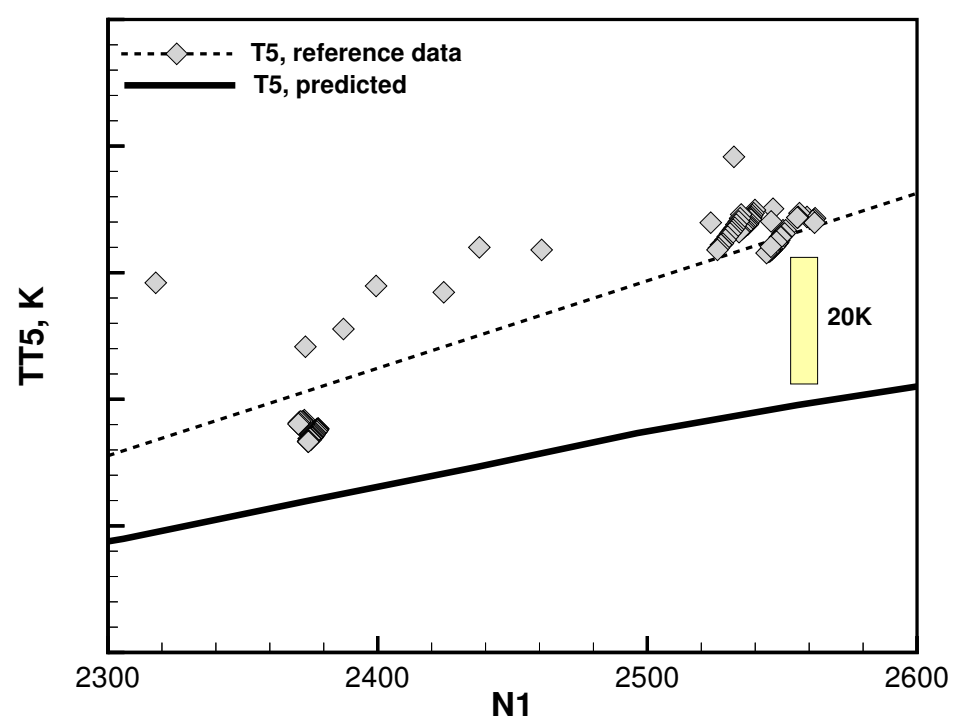

Figure 2: Engine Trent 900, simulated TT5 temperature and comparison with reference data from Rolls-Royce tests. 


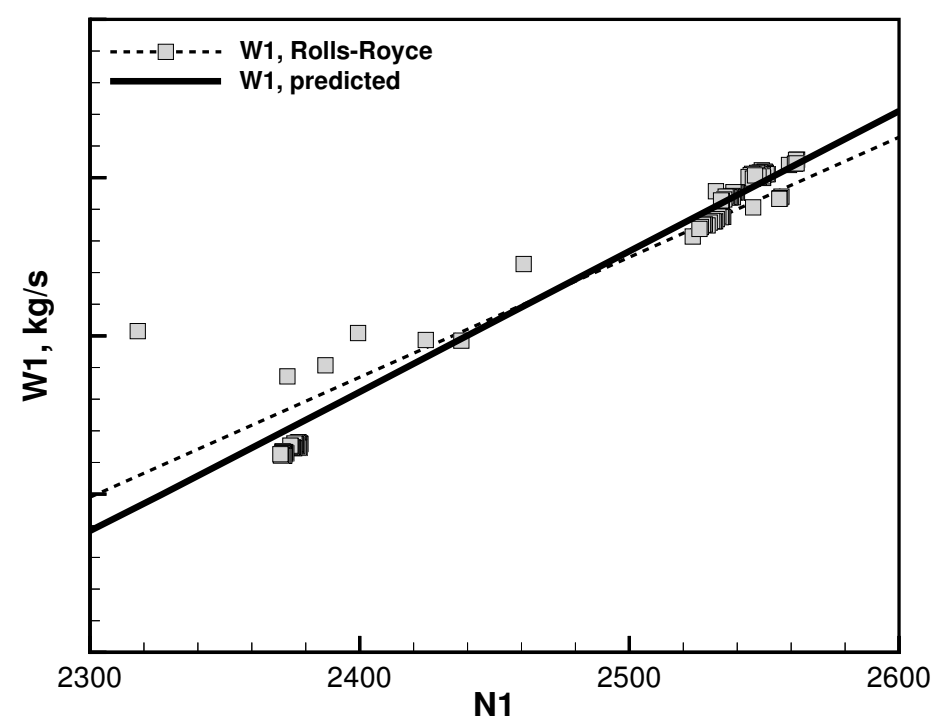

Figure 3: Engine Trent 900, simulated W1 mass flow and comparison with reference data from Rolls-Royce tests.

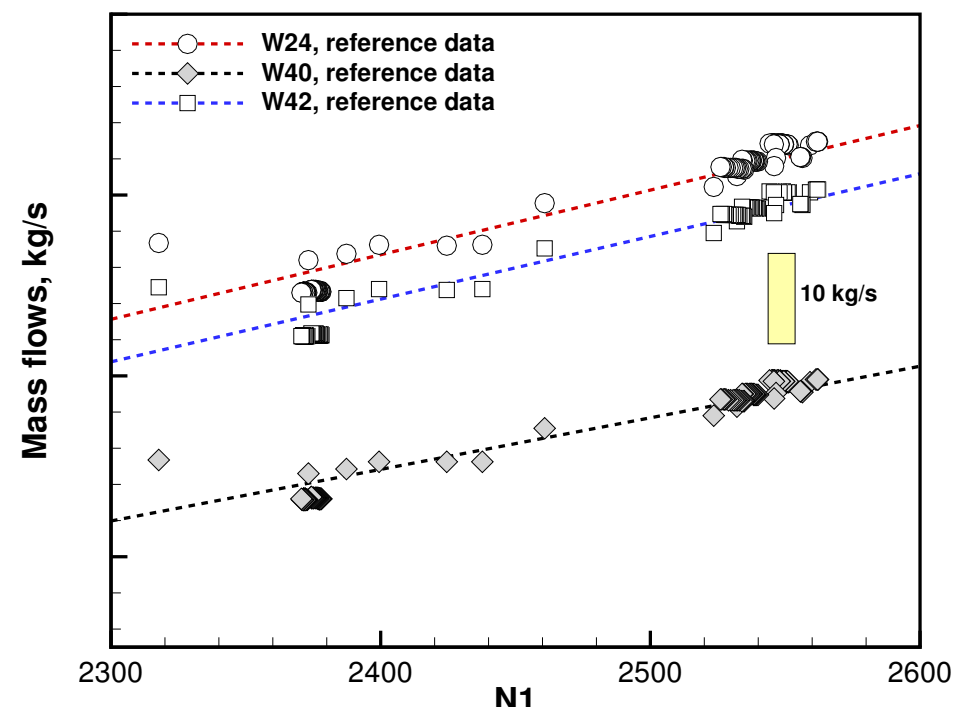

Figure 4: Engine Trent 900, simulated W4.0 mass flow and comparison with reference data from Rolls-Royce tests. 


\subsection{Dust Mass Loading Model}

The dust data was retrieved from the European Centre for Medium Range Weather Forecasts (ECMWF) near real time Copernicus Atmosphere Monitoring Service (CAMS). CAMS is a development of the Monitoring Atmospheric Composition and Climate (MACC) reanalysis ${ }^{17}$. CAMS tracks anthropogenic emissions, biomass burning and several atmospherically relevant chemical species. In addition, CAMS also uses an aerosol scheme described in full in Morcrette et al ${ }^{18}$. The CAMS global forecasting system has a T511L60 resolution which gives a horizontal resolution of approximately $40 \mathrm{~km}$ on a reduced-Gaussian grid, the vertical grid has 60 pressure levels from the surface to the top of the atmosphere. The horizontal resolution was upgraded during June 2016 and to maintain consistency any events earlier than July 2016 were removed from our analysis. By way of example, the dust mass loading data for March 2017 are presented in Figure 5. Mass loading is expressed as a mass mixing ratio, or unit mass of dust to unit mass of air.
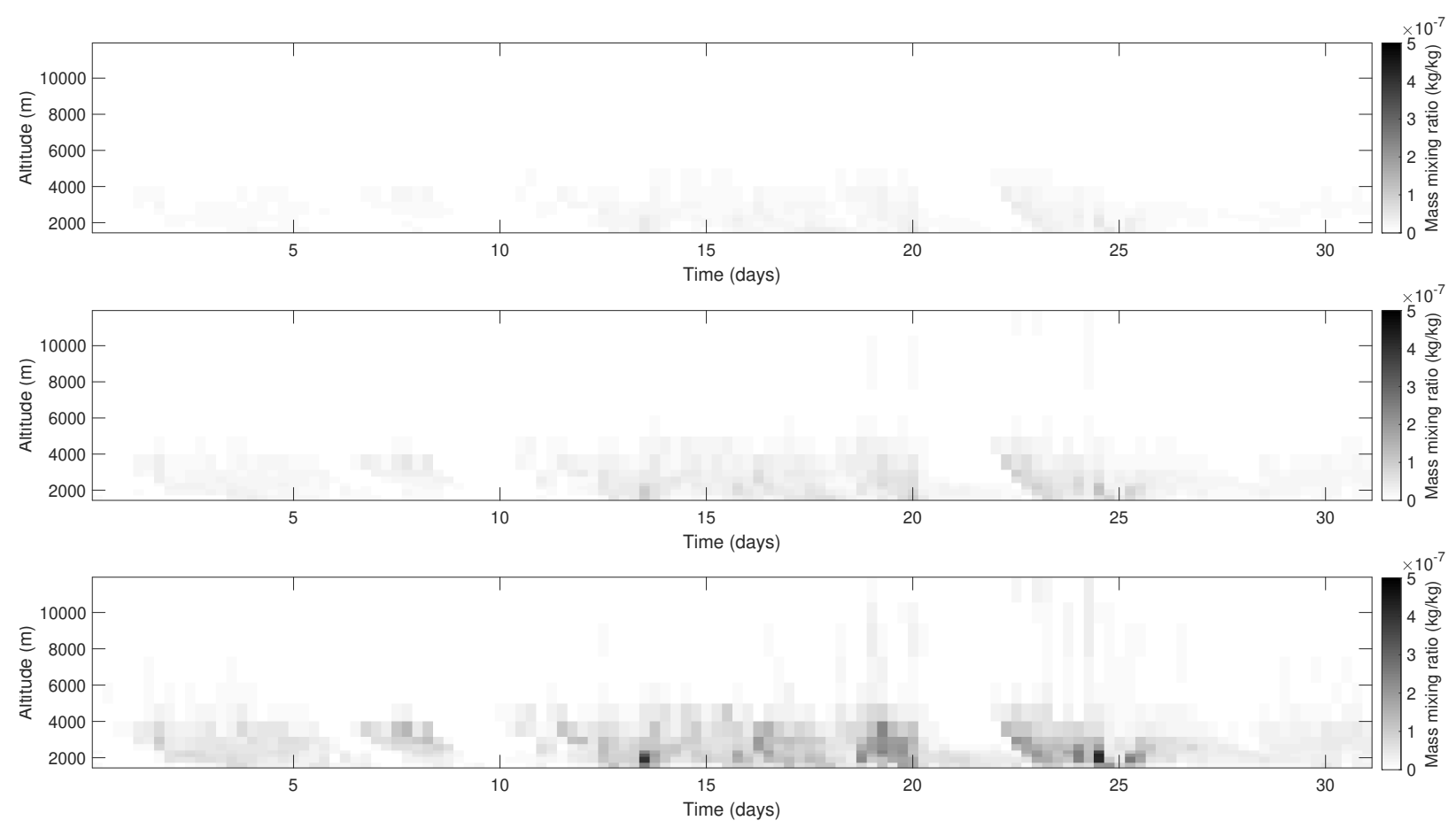

Figure 5: Example of a 31-day period with dust loading and its variation with altitude: Dust data retrieved from CAMS for March 2017 at Doha International Airport. Data presented in size bins: viewing from top to bottom: small $(0.03-0.55 \mu \mathrm{m})$, medium $(0.55-0.9 \mu \mathrm{m})$, and large $(0.9-20 \mu \mathrm{m})$.

The dust scheme has three bins: small $(0.03-0.55 \mu \mathrm{m})$, medium $(0.55-0.9 \mu \mathrm{m})$ and large $(0.9-$ $20 \mu \mathrm{m}$ ). The primary dust sources present in CAMS are the Sahara and Arabian deserts (see Figure 5 of Morcrette et $a l^{18}$ for details). Dust emission into the atmosphere is dependent on the wind-speed at the surface and removal is through dry or wet deposition, with some larger particles susceptible to gravitational settling. The temperature and horizontal wind speeds are assimilated 
from the ECMWF-IFS (Integrated Forecasting System) model and are provided from the same service to ensure consistency.

\section{Theory \& Calculations}

\subsection{Dust Ingestion}

The rate of dust ingestion into the engine can be determined by combining the dust mass loading (mixing ratio) and mass flow rate at the relevant engine stage. To estimate the engine mass flow rate we determine the engine requirements at each point along a flight trajectory. The trajectory considered includes taxi-out, take-off and climb-out to 9,000 m, with terminal point "1". At each time step, several engine parameters are calculated using FLIGHT, including the engine mass flow rate. If $c=c(x, t)$ is the dust concentration evaluated at a point in space and time from the ECMWF-CAMS retrievals, the dust mass ingestion rate into the fan stage is given by:

$$
\dot{m}_{p}=W_{1} c
$$

where $W_{1}$ is mass flow rate into the fan. Since FLIGHT assumes an international standard atmosphere, and since we only interrogate the dust mass loading data from ECMWF-CAMS, it is possible that local variations in barometric pressure are not accounted for. However, we believe that the coarseness of the grid would mask any effect that this would have on the results. The dust mass ingestion rate into the core is given by:

$$
\dot{m}_{p, c}=(1-\beta) W_{1} c=W_{2.1} c
$$

where $W_{21}$ is mass flow rate into the intermediate pressure compressor and $\beta$ is the bypass ratio factor. The concentration reduction effect due to centrifuging effect of the fan is neglected in the absence of a generalised model and lack of information on the dust inertial properties. The entry dust mass $C_{1}$ for a given flight is calculated by integrating the dust dose along the trajectory:

$$
C_{1}=\int_{o}^{1} W_{1} c d t
$$

whilst the core dust is calculated from:

$$
C_{1, c}=\int_{o}^{1}(1-\beta) W_{1} c d t
$$

\subsection{Dust Deposition}

The generalised deposition model by Kern \& Seaton ${ }^{19}$ for application to heat exchanger fouling is applied to estimate the timescales involved in deposit build-up and performance deterioration. It assumes that the build-up of particle deposits on compressor blade rows is asymptotic, in which a deposition rate is tempered by a shedding rate that grows as a function of deposit mass. A similar 
model is proposed by Clarkson \& Simpson to describe the behaviour of deposit build-up on turbine nozzle guide vanes ${ }^{9}$. The rate of deposition per blade passage is thus:

$$
\begin{aligned}
\frac{d M}{d t} & =\frac{\dot{m}_{p, c}}{N_{b}} \chi \xi-\left(\frac{\dot{m}_{p, c}}{N_{b}} \chi \phi+\lambda\right) M \\
& =\frac{\dot{m}_{p, c}}{N_{b}} \chi(\xi-\phi M)-\lambda M
\end{aligned}
$$

where: $\dot{m}_{p, c}$ is the particle mass flow rate into the core determined from Eq. $2 ; N_{b}$ is the number of passages in the blade or vane row; $M$ is the total deposit mass in the blade or vane passage; $\chi$ is the particle-blade interaction probability, also known as the coefficient of particle transport, which depends upon the Stokes number ${ }^{20} ; \xi$ is the sticking probability of the deposit-free surface, which depends on the particle elastic and rheological properties ${ }^{12 ; 21}$ and surface condition; $\phi$ is the autoretardation coefficient, which accounts for the gradual decline of the particles' propensity to stick e.g. due to looser bonds in outer deposit layers ${ }^{22}$; and $\lambda$ is the shedding factor due to aerodynamic re-entrainment. On a macroscopic level, particle re-entrainment due to inertial collisions is not distinguishable from auto-retardation and is a function of particle concentration, therefore it is included in $\phi$ rather than $\lambda^{22}$. This one-dimensional model does not account for local variations in deposit thickness or the influence of end-wall vortices or cooling holes. The values of $\chi, \xi, \phi$ and $\lambda$ are difficult to determine analytically owing to the huge number of contributory independent variables, some of which cannot be measured at the relevant engine section. However, their values may be estimated empirically and used to determined the timescales of engine deterioration.

\subsection{Compressor Performance Deterioration}

We adopt the work of Döring et al. ${ }^{22}$ to the current work to relate the dust mass ingestion to engine compressor performance deterioration. Döring et al. ${ }^{22}$ applied the model of Kern \& Seaton to the fouling of a linear compressor cascade. They assumed that the aerodynamic re-entrainment (or shedding) term $\lambda$ was negligible when compared with the influence of auto-retardation and re-entrainment through inertial collisions term $\phi$, hence particle removal diminishes when concentration drops to zero, and $\lambda \approx 0$. This is an assumption that cannot be applied when the deposition rate is high, such as the case of a nozzle guide vane stage being overwhelmed by molten volcanic ash or mineral dust, as the deposit build-up narrows the throat and increases the aerodynamic shear force. It is this mechanism that is thought to be responsible for removing glassy deposits, post-exposure, during flight through clean air, a phenomenon that allowed engines to restart in some historical volcanic ash exposure events ${ }^{8 ; 9}$. The fouling of compressor blades happens over a much longer timescale and does not lead to a large scale build up of surface material. Neglecting $\lambda$, Eq. 5 can be transferred into the mass domain and written as:

$$
\frac{N_{b}}{\dot{m}_{p}} \frac{\mathrm{d} M}{\mathrm{~d} t}=N_{b} \frac{\mathrm{d} M}{\mathrm{~d} C_{1, c}}=\chi \xi-\chi \phi M
$$


so that the deposition process is a function of the total ingested dust mass into the core $C_{1, c}$ as given in Eq. 4 and independent of the current particle concentration and engine mass flow rate. Solving Eq. 6 gives the asymptotic deposit mass model:

$$
M=\frac{\xi}{\phi}\left(1-e^{-\chi \phi t}\right)=M_{\infty}\left(1-e^{-\frac{C_{1, c}}{N_{b} \tau}}\right)
$$

and $\tau$ is the time constant in the mass domain, with units of mass. This model assumes that the empirical factors $\chi, \xi$, and $\phi$ are independent of engine mass flow, which is unlikely to be true in practice. However, transferring the model into the mass domain allows a first order estimation of the performance deterioration when the concentration and mass flow rate are different.

A final assumption of Döring et al.'s approach is that the deterioration of performance parameters is also asymptotic, i.e.:

$$
\Delta \psi=\Delta \psi_{\infty}\left(1-e^{-\frac{C_{1, c}}{N_{b} \tau}}\right)
$$

where $\psi$ is the static pressure rise coefficient, and:

$$
\Delta \omega=\Delta \omega_{\infty}\left(1-e^{-\frac{C_{1, c}}{N_{b} \tau}}\right)
$$

where $\omega$ is the total pressure loss coefficient. The asymptote thus represents the maximum loss in performance due to deposition at a given operating point, while the time constant controls the rate of performance deterioration.

\subsection{Equivalent Dose}

For the sake of consistency with the existing literature and parlance in industry, we will use the term dose to refer to product of the concentration by volume and the duration of exposure, adopting the units gs $/ \mathrm{m}^{3}$. Accordingly, we can calculate the dose for the current case and compare it to the safe-to-fly limit. However, to distinguish the current case of normal operations from a non-benign airport, from the potential flight safety risk of an encounter with a volcanic ash cloud, we will adopt the term equivalent dose. Since the engine mass flow rate and air density are variant along all points of the trajectory, the dose for a given flight, $\delta_{1}$ is calculated by integrating the volume flow rate along the trajectory and dividing by the ingested dust mass. Since the mixing ratio of the dust particles is $\sim 10^{-7}$, their contribution to the gas density can be neglected, and the concentration by volume simply introduced:

$$
\delta_{1}=\frac{\int_{o}^{1} W_{1} c d t}{\int_{o}^{1} W_{1} / \rho_{g} d t}=\int_{o}^{1} \rho_{g} c d t \equiv \int_{o}^{1} c_{v} d t
$$

where $\rho_{g}$ is the gas density at each point along the trajectory and $c_{v}$ is the dust concentration by volume. It can be seen that the term on the right hand side is indeed the product of the exposure duration and dust concentration, as used by Clarkson et al. ${ }^{8 ; 9}$ and Prata et al. ${ }^{10}$, provided that the concentration is expressed in units of $\mathrm{g} / \mathrm{m}^{3}$. We will use this method to calculate the dose 
encountered during the flights simulated in this study, to allow comparison with the values discussed in Section 1.

\section{Results}

An Airbus A380-841 with Rolls-Royce Trent 900 turbofan engines is required to fulfil a transport mission from Doha (Qatar) to London Heathrow (UK). The great-circle distance is $\sim 5,300 \mathrm{~km}$ ( $\sim 2,860$ nautical miles). The weight is estimated assuming full passenger load, with the necessary fuel requirements to fly this route. Dust ingestion accounting is terminated when the aircraft reaches an altitude of 9,000 $\mathrm{m}(\sim 29,500$ feet). The ECMWF-CAMS forecast is updated every 6 hours, so we have simulated four flights per day in order to understand how the departure time affects the dust ingestion. Tracking the operations over a full month requires the simulation of about 120 flights. In this sensitivity study, three 31-day periods that contained high dust loads were selected for further analysis. These three periods were March 2017, March 2018 and 15-May to 14-June 2017, with each showing a considerable amount of dust in the atmosphere.

\subsection{Diurnal variation of dust ingestion}

Dust ingestion simulation results are presented in Figures 6-8. Dust data labelled as light, medium and heavy refer to the average particle size. Light dust has particles diameter $d=0.03-0.55 \mu \mathrm{m}$; medium dust has $d=0.55-0.90 \mu \mathrm{m}$, and heavy dust $d=0.90-20.0 \mu \mathrm{m}$.

Figures 6, 7 and 8, show that there are four instances of large dust ingestion over the three periods studied, with core dust estimated as 20 to $35 \mathrm{~g}$ in at least 5 flights. The corresponding total dust ingestion into the fan (all particles sizes) is one order of magnitude higher, scaling roughly with the by-pass ratio of this engine. Dust data from 15 May to 14 June 2017, Figure 7, do not show equally severe cases of dust ingestion, but at least in two instances the core dust mass ingested is $\sim 20 \mathrm{~g}$.

Finally, the case of March 2018, Figure 8, shows the same levels of severity of the previous year, with a particularly bad day towards the end of the month, when core dust is estimated as 36 $\mathrm{g}$, out of a total ingestion of $270 \mathrm{~g}$ across all particle sizes.

Table 1: Summary of dust mass ingestion simulations. A total of 4 flights per day over each month were simulated. All data in $[\mathrm{kg}]$. Averages are calculated across all flights.

\begin{tabular}{lcccccc}
\hline & $C_{\max }$ & $C_{c, \max }$ & $C_{c, \min }$ & $\bar{C}$ & $\bar{C}_{c}$ & $\sigma_{c}$ \\
\hline March 2017 & 0.439 & 0.037 & 0.001 & 0.1025 & 0.0086 & 0.0070 \\
May-June 2017 & 0.249 & 0.020 & 0.002 & 0.0921 & 0.0077 & 0.0042 \\
March 2018 & 0.423 & 0.036 & 0.001 & 0.1078 & 0.0091 & 0.0061 \\
\hline All months & - & - & - & 0.1008 & 0.0085 & 0.0059 \\
\hline
\end{tabular}

Table 1 shows a summary of data for the worst dust days, along with average values of all 


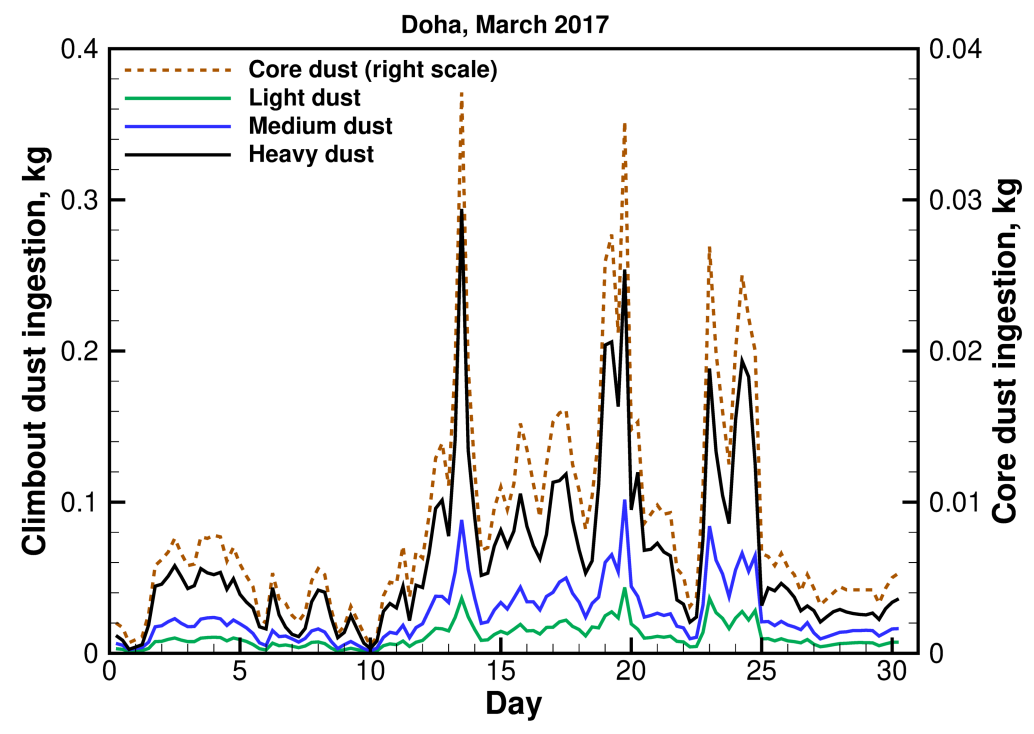

Figure 6: Simulation of dust ingestion of an Airbus A380-841 flying out of Doha, March 2017.

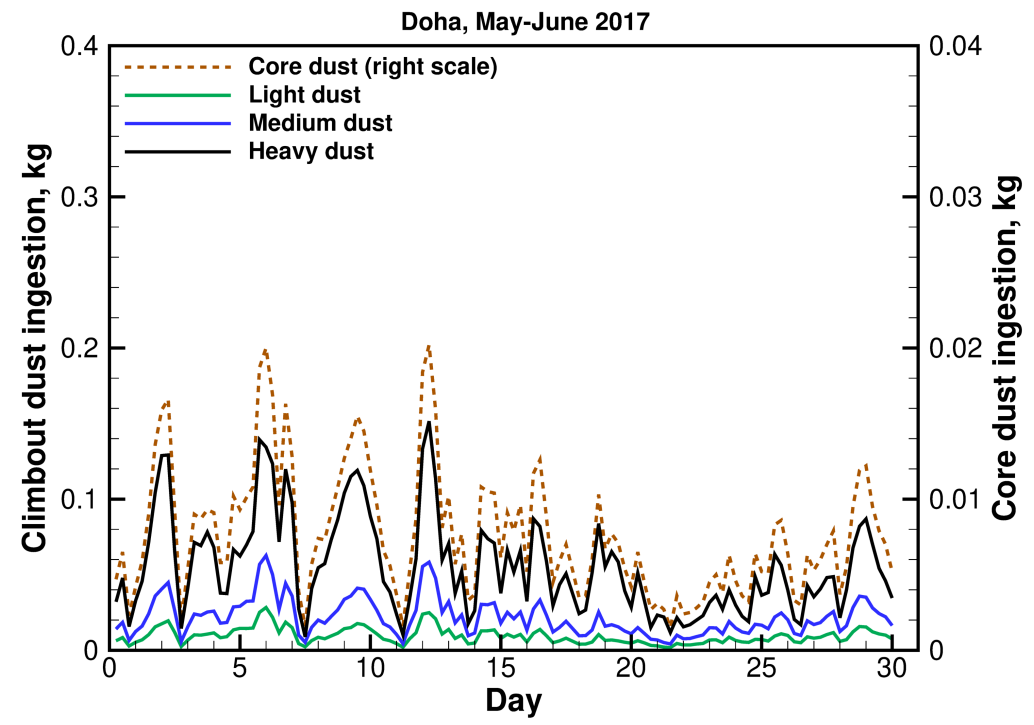

Figure 7: Simulation of dust ingestion of an Airbus A380-841 flying out of Doha, May-June 2017.

dust ingestion and core dust ingestion. The three columns to the right are statistical data over the entire number of flights per month $(\sim 120)$. We conclude that the average core dust is $\sim 8.5$ $\mathrm{g}$, and the total dust into the fan is $\sim 100 \mathrm{~g}$. 


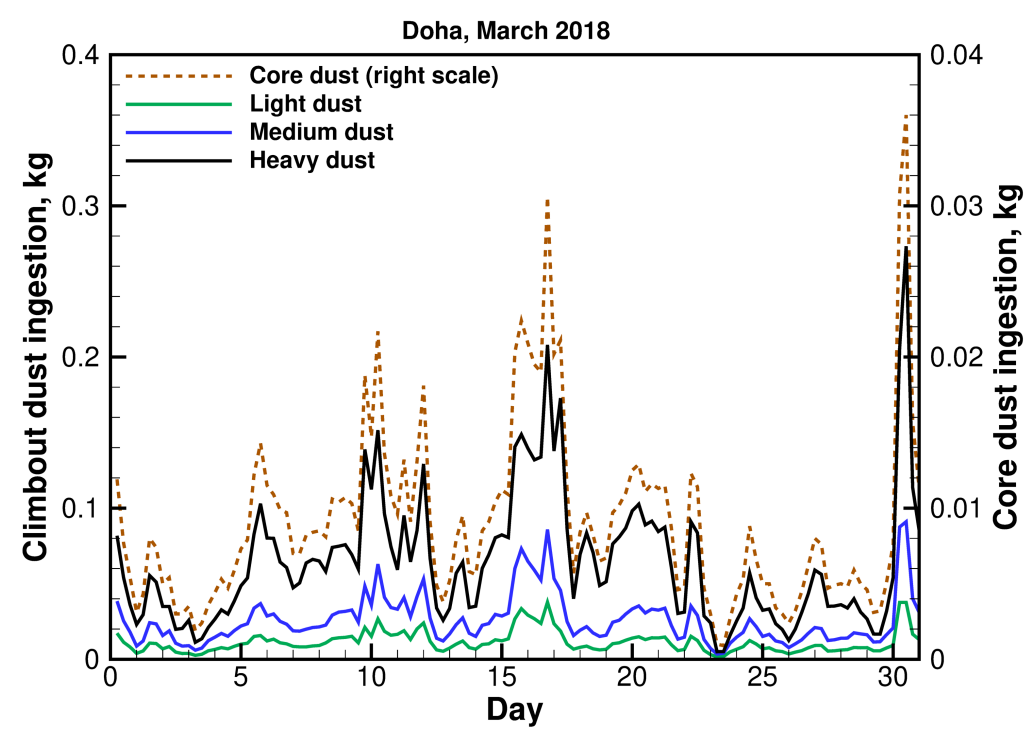

Figure 8: Simulation of dust ingestion of an Airbus A380-841 flying out of Doha, March 2018.

\subsection{Dust mass ingestion during dust storms}

We select a single departure during a dust storm to investigate the dust ingestion rate as a function of flight time. Figure 9 shows the climb-out trajectory of an A380-841 departing from Doha at 08:00 on 14 March 2017. The climb-out trajectory is composed of 8 segments, listed in Table 2 . The aircraft reaches the initial cruise altitude (ICA) of 36,000 feet after approximately 27 minutes. The mixing ratio varies between a minimum of $\sim 0.3 \mathrm{mg} / \mathrm{kg}$ to a maximum of $0.5 \mathrm{mg} / \mathrm{kg}$ of air, up to an altitude of around 20,000 feet (Flight Level 200). The concentration level exhibits as discontinuous and variable, although the use of a more refined grid would smooth this. Mineral dust concentrations vary with altitude, typically decreasing as the source is at the surface and deposition effects (gravity, precipitation) remove dust with time. However horizontal transport from remote areas combined with low advection of surface dust can create conditions where dust levels are actually higher with altitude, see Day 11 on Figure 5 for an example.

The transition from one segment into another is characterised by a change in engine mass flow rate, also shown in Figure 9; the FLIGHT software does not simulate engine transients. The engine mass flow peaks at two moments during the climb-out: firstly during the take-off run, at around $1,100 \mathrm{~kg} / \mathrm{s}$; and secondly as the aircraft enters the second climb segment to 10,000 feet altitude, where the mass flow increases to over $1,000 \mathrm{~kg} / \mathrm{s}$. At this point, there is still a substantial concentration of dust in the atmosphere.

Figure 10 presents parameters relating to the engine core. Reflecting the peaks in engine mass flow rate, the turbine entry temperature (hottest gas in the engines), is highest during take-off for a period of around 30 seconds until the thrust is cut back, at which point the temperature falls to $\sim 1,500 \mathrm{~K}$. The temperature rises again to $\sim 1,650 \mathrm{~K}$ when the second climb segment is initiated. 
The ingestion of dust continues for approximately 5 minutes' flight time, as can be seen in the plot of cumulative dust mass.

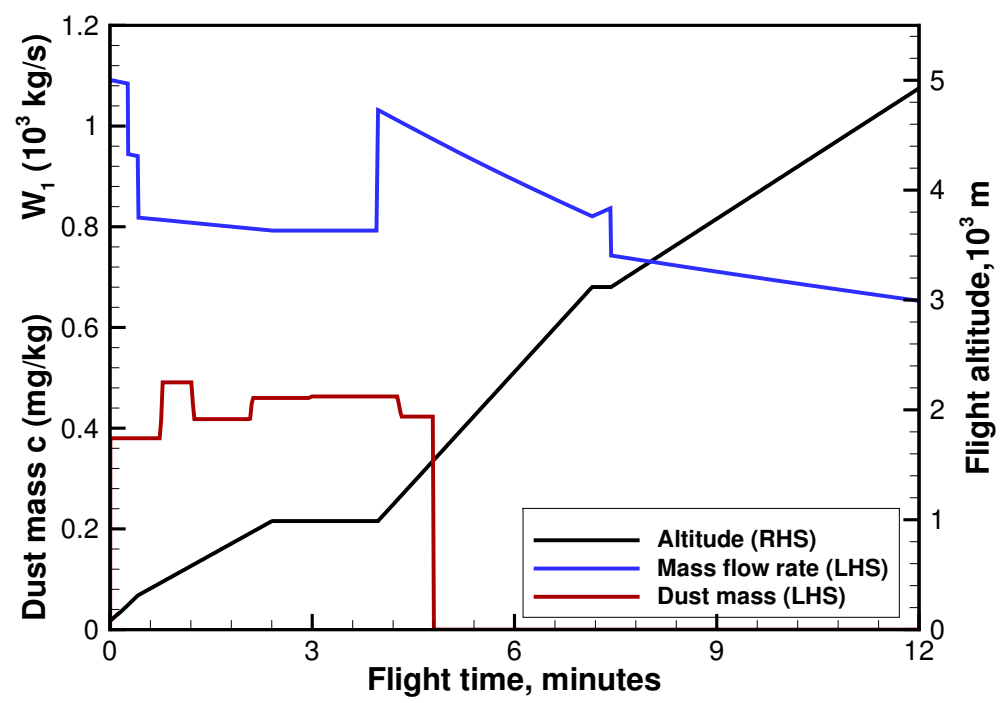

Figure 9: Simulated fan stage mass flow $\left(W_{1}\right)$, dust mass mixing ratio, and altitude as a function of flight time, of an Airbus A380-841 climbing out from Doha at 08:00 on 14 March 2017.

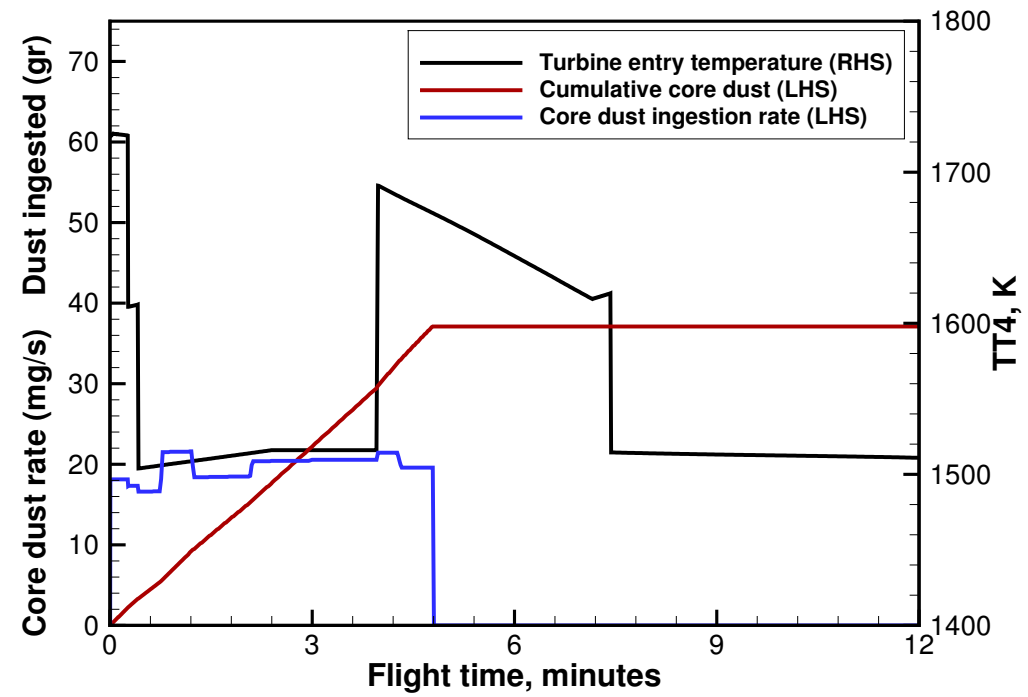

Figure 10: Cumulative dust mass ingested into the core, core dust ingestion rate, and turbine entry temperature as a function of flight time, of Airbus A380-841 engine climbing out from Doha at 08:00 on 14 March 2017.

\subsection{Dust mass ingestion while holding on a stack}

An aircraft holding on a stack before landing has been simulated, with the aircraft assumed to hold for 20 minutes at 10,000 feet, $10 \mathrm{~nm}$ out of Doha, at GPS coordinates 25.479 N, 51.94 E (this 
is in the Persian Gulf). It is demonstrated that dust ingestion into the engine core would be $\sim 8$ $\mathrm{g}$, almost as much as in the worst conditions shown in the climb-out analysis.

\section{Discussion}

The results show quite a large fluctuation in the total dust mass ingested. Departures on dust-days are identified by the highest peaks, in particular during March 2017, but peaks occur approximately every two to three days indicating that ingestion rates may be high even during normal operations.

\subsection{Equivalent Compressor Deterioration Rate}

To examine the effect of multiple dust-day operations on engine performance, we implement the maximum, average, and minimum per flight ingested dust mass to the deterioration model of Döring et $a l .{ }^{22}$. The particle mass per blade passage is calculated based on the number of stator vanes of the second stage high pressure compressor of the Trent 900, estimated to number 64 . The three results are presented in Figure 11, alongside the validation case presented by Döring et al. ${ }^{22}$. The mixing ratio on a dusty day at Doha is approximately eight times greater than the estimated particle background atmospheric concentration at Barcelona El Prat airport.

The vertical line labelled $C_{1, c}^{*}$ represents the compressor performance deterioration time constant, $\tau$, borrowed from Döring et al. ${ }^{22}$. This quantity $(4.1 \mathrm{~g})$ represents the total mass accumulated per blade passage when the performance loss has reached $\sim 63 \%$ of the asymptotic value. It is based on an engine running at ICAO taxi/idle static sea level condition in a high moisture content environment. This equates to $\sim 250$ flight cycles, based on the standardized ICAO landing and take-off (LTO) taxi/idle cycle time in a concentration of $0.04 \mathrm{mg} / \mathrm{kg}$. The intersections of the ' $C_{1, c}^{*}=\tau^{\prime}$ ' and the 'min./ave./max. concentration' lines in Figure 11 correspond to the number flight cycles it would take to for the engine to ingest the $4.1 \mathrm{~g}$ of dust per blade passage. For average conditions in Doha this equates to $\sim 31$ climb-out cycles.

This finding relies on the assumption that differences in concentration and stage Reynolds number have no bearing on the deterioration rate, and is based on the "high moisture setup" in Döring et al. ${ }^{22}$. This is unlikely to be true, but nevertheless illustrates how the methodology in the current work could be used to optimise compressor wash scheduling. The coupled weather-flight performance code computes a trajectory simulation in approximately 10 minutes; dust dispersion forecasts are issued every 6 hours. If aircraft weight data are known, this methodology could be implemented into maintenance scheduling planning software to afford real-time estimations of onwing maintenance operations such as compressor washing. However, more rig tests are required to determine the deterioration time constants in order to improve the prediction accuracy, including deposition tests on the other major fouled engine sections such as the high pressure turbine. 


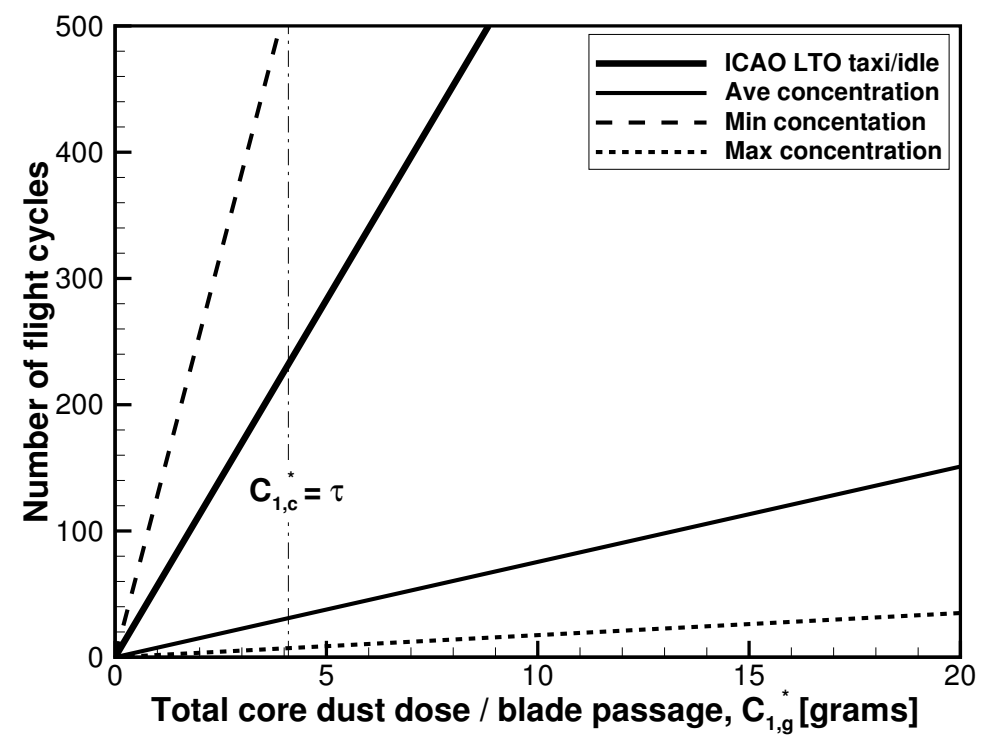

Figure 11: Number of equivalent flight cycles out of Doha as a function of core dust mass ingested into the compressor. The vertical line represents the time constant of the asymptotic deterioration rate determined experimentally in Döring et al ${ }^{22}$.

\subsection{Equivalent Dose}

The cumulative dust ingestion mass has been calculated for each segment of the climb-out and is presented in Table 2. The total mass during the climb-out, and the dust mass ingested whilst holding on a stack is also shown. The time-averaged concentration by volume has been calculated and combined with the duration of exposure to calculate the equivalent dose. The largest dose during the climb-out was found to occur during the climb at constant calibrated airspeed to 3,000 feet. This dose level, of $0.184 \mathrm{gs} / \mathrm{m}^{3}$ is two orders of magnitude less than the incidents that were classed as "safety critical" by Clarkson \& Simpson et al. ${ }^{9}$. This segment lasts approximately two minutes but through a relatively high dust concentration of over $1.5 \mathrm{mg} / \mathrm{m}^{3}$. It is worth noting that the average dust concentration (by volume) calculated for each climb out segment is of the order $1-2 \mathrm{mg} / \mathrm{m}^{3}$. This is of the same order of magnitude as the concentration corresponding to low visibility during dust storms, as discussed in Section 1.

Ordinarily, as noted by Döring et al. ${ }^{22}$, the aircraft would experience the highest ingestion rate of dust during taxi and take-off, i.e. at ground level where the ambient concentration is greatest. However, based on the simulations we have conducted, it is possible that the dust deposits observed in engines operating from airports such as Doha Airport are ingested both during taxi and climbout. This is important, because analysis of airport dust types is generally based on samples taken at ground level. It is likely that the chemical composition (and therefore sticking ability) of dust at higher altitudes is different to that on the ground, owing to the differences in mineral proportion occupied by different particle size bands ${ }^{23}$. This reinforces the point that in-situ ground 
Table 2: Calculation of dose for each segment of climb-out from Doha International Airport, 0800 14 March 2017.

\begin{tabular}{lrrcc}
\hline Flight segment & $\begin{array}{r}\Delta t \\
{[\mathrm{sec}]}\end{array}$ & $\begin{array}{r}\Delta C_{\text {all }}[\mathrm{g}] \\
{\left[\mathrm{mg} / \mathrm{m}^{3}\right]}\end{array}$ & $\begin{array}{c}\overline{c_{v}} \\
{\left[\mathrm{gs} / \mathrm{m}^{3}\right]}\end{array}$ \\
\hline Taxi to break release & & & & \\
Accelerate to KCAS1 & 7.3 & 12.1 & 1.809 & 0.013 \\
Accelerate to TAS $>$ V2 + 20 kt & 7.9 & 12.9 & 1.768 & 0.014 \\
Climb to 800 ft & 9.1 & 13.3 & 1.583 & 0.014 \\
Thrust cutback, climb to 3,000 ft & 119.8 & 169.7 & 1.538 & 0.184 \\
Level acceleration to 250 KCAS & 91.3 & 136.0 & 1.618 & 0.148 \\
Climb at constant KCAS to 10,000 ft & 192.2 & 90.0 & 0.508 & 0.098 \\
Level acceleration to 273 KCAS & 16.9 & 0 & 0 & 0 \\
Climb at constant to optimum ICA & 1189.2 & 0 & 0 & 0 \\
\hline Total & 1633.7 & 434.0 & 0.288 & 0.471 \\
\hline Holding on a stack, 10,000 ft. & $1,200.0$ & 8.0 & 0.410 & 0.492 \\
\hline
\end{tabular}

measurements taken at the airport may not necessarily provide all the mineralogical information required to anticipate the maintenance burden of a particular route.

Furthermore, the second peak dust ingestion mass flow rates of $22 \mathrm{mg} / \mathrm{s}$ coincides with the peak turbine entry temperature of 1,700 K, as shown in Figure 10. While the composition of the dust ingested during climb-out is not known, the peak temperatures are higher than the melting points of several of the dust samples from various locations in the Arabian peninsula reported by Krisak ${ }^{5}$, which exhibit melting points of 1,450,1,520, and 1,570 K. At these temperatures, CMAS glass is able to form from the molten products on turbine vane and blade surfaces, potentially leading to degradation of the thermal barrier coating through cycle-driven spallation. As aircraft engine core temperatures continue to increase, the dust concentrations experienced during a climb-out on a dusty day may one day attract the same attention as the risk posed by volcanic ash.

High concentration, low duration exposure events are seen to result in more severe damage than their converse, possibly due to a non-linear rise in deposition rate with dust concentration ${ }^{24 ; 9}$. For a climb-out lasting 27 minutes, the dose is $0.471 \mathrm{gs} / \mathrm{m}^{3}$. For a hold lasting 20 minutes, the dose is calculated to be $0.492 \mathrm{gs} / \mathrm{m}^{3}$. To exceed the safe-to-fly limit of $14.4 \mathrm{gs} / \mathrm{m}^{3}$, the average ambient dust concentration would have to exceed an average of $8.9 \mathrm{mg} / \mathrm{m}^{3}$ during climb-out, and $12 \mathrm{mg} / \mathrm{m}^{3}$ during a hold. This is almost six times the peak concentration we have calculated along any climbout trajectory. Therefore, we conclude that flight safety is unlikely to be compromised during a single flight due to mineral dust ingestion. However, if the dose approach is applied to multiple exposure events in dusty environments, the dose limit would be reached within 31 climb-out cycles, or 35-40 cumulative hours in holding patterns. 


\subsection{Dust Flight Concept}

The term dust-day has been used in the atmospheric science literature to define a level of ambient dust, as discussed in Section 1. We stop short of using this definition to categorise a dust-day in the context of flight operations for three reasons. The first is connected to the discussion on dose, in that it is equally important to consider the duration of exposure when assessing the potential damage to engines, which a single concentration value, measured at the airport, would not capture. The second is that, as we have shown Table 2, the mean concentration at the airport may differ considerably along the climb-out flight path, depending on the prevailing conditions and the chosen flight path. The third is that a concentration-based dust-day at one airport may look like a standard day at another.

Instead we explore a mission-based approach to categorise operations in which large quantities of dust are expected to be ingested. A flight which ingests a high quantity of dust could be termed a dust-flight in shorthand. We elect to not specify a dose threshold for this, as has been adopted for a safe-to-fly limit during volcanic ash encounters, because the longer-term damage caused by multiple small dose events will manifest differently from one engine design to the next. While dose is a helpful comparator for dust exposure events, its use as a dust-flight definition would lose some of the nuances afforded by analysing dust exposure on a flight-by-flight basis.

Instead, we propose that a dust-flight be based on an operational mean dust mass ingested for a given city pair/aircraft combination. This would allow a connection to be made to engine deterioration rate and maintenance scheduling, as discussed in Section 5.1, or may allow for the prediction of unscheduled maintenance. Using the statistical data from all 365 simulations, given in Table 1 , by way of example we flag any simulation in which $C_{c}>\left(\bar{C}_{c}+2 \sigma_{c}\right)$ as a dust-flight. This equates to a threshold of $20.2 \mathrm{~g}$ ingested into the core in one flight, for an A380-841 flying from Doha to London Heathrow. The data in Table 3 show that a similar proportion of flights in March 2017 and 2018 are categorised as dust-flights, but we see that no flights are flagged such in May-June 2017. This is reflected in the height of the peaks relative to the peaks shown in March 2017 and March 2018, visible in Figure 6-8. The single departure analysed in Section 4.2 is one of these. We also see that in each month the proportion of flights exceeding the mean falls below $50 \%$, which indicates that the median dust mass ingested is less than the mean. Note that the simulation of holding on a stack did not meet our criterion to be categorised as a dust-flight.

Table 3: Proportion of simulation results categorised as dust-flights, and share of total mass ingested during dust-flights, shown for March 2017, May-June 2017, and March 2018.

\begin{tabular}{cccc}
\hline Time period & March 2017 & May-June 2017 & March 2018 \\
\hline Proportion designated as dust-flights [\%] & 6.6 & 0.0 & 6.5 \\
Share of total mass ingested [\%] & 21.3 & 0.0 & 18.5 \\
\hline
\end{tabular}

Since the threshold in this study has its basis in statistics, it is perhaps unsurprising that 
almost $95 \%$ of the flights lie within two standard deviations of the mean. However, if we calculate the share of the monthly total dust ingested contributed by these flights, we notice that just a few dust-flights can account for $\sim 20 \%$ of the burden. While such dust ingestion events are unlikely to necessitate off-wing maintenance, flagging the occurrence of a dust-flight could help to prompt a boroscope inspection of an engine.

Finally, given that in at least one the dust-flights in March 2017, discussed in Section 5.2, dust continued to be ingested up to 2,000 $\mathrm{m}$ altitude, this analysis reinforces the point that the concentration and composition of the majority of the dust an aircraft engine ingests may not be reflected in-situ measurements taken at the airport. A mission-based approach may therefore be more suitable for designating an airport as non-benign, and ultimately, for determining engine durability in dusty environments.

\section{Conclusions}

Dust storms are relatively frequent in certain parts of the world, with events lasting from a few hours to a few days. These events may cause disruption to flight operations, and medium- to long-term damage to the engines, with consequent increase in the direct operating costs.

A methodology was presented which couples a flight performance code with an open source real-time weather forecast tool, to predict the total dust mass ingested by an aircraft engine during climb-out from a high-traffic airport in the Middle East. The engine mass flow requirements were determined using the FLIGHT software, and the dust concentration was retrieved using reanalysis datasets produced by ECMWF-CAMS. The maximum dust mass ingested into the core during a single flight was just under $40 \mathrm{~g}$.

The results are heavily dependent on the resolution of the dust data and on the grid, which in this case was $40 \mathrm{~km} \times 40 \mathrm{~km}$. With the data considered, a 6-hour delay at the airfield would greatly reduce the amount of dust ingestion, although this option cannot be extrapolated to all possible events.

The average dust mass ingested per flight was shown to exceed that predicted in prior work. Using a pre-existing deterioration model, we conclude that this would lead to a reduction in the time between compressor wash intervals, although more experimental data is required to investigate the effect of blade Reynolds number on total pressure loss and static pressure rise reduction. The deterioration rate of the high pressure turbine stage was not estimated here, but the methodology could be extended to include it and other sections of the engine using the engine parameter outputs of the flight performance code.

A single, worst case flight was singled out for comparison with other dust exposure events in the literature. We conclude that dust exposure during a single flight in a dust storm is unlikely to pose a risk to flight safety based on the dose approach and predictions of the peak concentration along the trajectory. However, the dust levels in the Middle East were predicted to reduce the 
deterioration time constant by roughly 8 times, leading to $95 \%$ of the asymptotic performance loss being reached after 80 climb-out cycles in average Doha conditions, rather than the 800 cycles as predicted for operations in and out of Barcelona. This may predicate the need for more regular compressor washes of aircraft engines operating in the Middle East.

A new approach to categorise a high dust ingestion flight is suggested, termed a dust-flight. This may be used to categorise an aircraft/city pair combination, rather than just an airport, as non-benign. The methodology as a whole has the potential to be used to optimise on-wing maintenance schedules in dusty environments, and could be used to prompt boroscope inspections or predict unscheduled maintenance when coupled with meteorology forecasts.

\section{Acknowledgements}

The authors wish to thank Rolls-Royce for the permission to validate our engine models against real data. Data were sanitised for reasons of confidentiality. This work contains data Generated using Copernicus Atmosphere Monitoring Service Information (2019). Neither the European Commissionnor ECMWF is responsible for any use that may be made of the information it contains. Dr Parkes is the recipient of the Ekpe Research Impact Fellowship in the Department of Mechanical, Aerospace and Civil Engineering at The University of Manchester.

\section{References}

[1] Airbus. Global Market Forecast 2018-2037.

[2] Lancaster N. Aeolian Processes. Reference Module in Earth Systems and Environmental Sciences, Jan 2014. DOI: 10.1016/B978-0-12-409548-9.09126-0.

[3] Tanaka TY and Chiba M. A numerical study of the contributions of dust source regions to the global dust budget. Global and Planetary Change, 52(1-4):88-104, Jul 2006. DOI: 10.1016/J.GLOPLACHA.2006.02.002.

[4] Notaro M, Yu Y, and Kalashnikova OV. Regime shift in Arabian dust activity, triggered by persistent Fertile Crescent drought. Journal of Geophysical Research: Atmospheres, 120(19):10,229-10,249, Oct 2015. DOI: 10.1002/2015JD023855.

[5] MB Krisak. Environmental Degradation of Nickel-Based Superalloys Due to Gypsiferous Desert Dusts. PhD thesis, Air Force Institute of Technology WPAFB, 2015.

[6] A.M. Al-Dousari and J. Al-Awadhi. Dust fallout in northern Kuwait, major sources and characteristics. Kuwait Journal of Science ES Engineering, 39(2A):171-187, 2012.

[7] Shao Y, Yang Y, Wang J, Song Z, Leslie LM, Dong Ch, Zhang Z, Lin Z, Yutaka K, Yabuki S, and Chun Y. Northeast Asian dust storms: Real-time numerical prediction and validation. 
Journal of Geophysical Research: Atmospheres, 108(D22):2003JD003667, Nov 2003. DOI: 10.1029/2003JD003667.

[8] Clarkson RJ, Majewicz EJE, and Mack P. A re-evaluation of the 2010 quantitative understanding of the effects volcanic ash has on gas turbine engines. Proceedings of the Institution of Mechanical Engineers, Part G: Journal of Aerospace Engineering, 230(12):2274-2291, Oct 2016. DOI: $10.1177 / 0954410015623372$.

[9] Clarkson R and Simpson H. Maximising Airspace Use During Volcanic Eruptions : Matching Engine Durability against Ash Cloud Occurrence. In STO-MP-AVT-272, pages 1-20. NATO STO, 2017. DOI: 10.14339/STO-MP-AVT-272.

[10] Prata AT, Dacre HF, Irvine EA, Mathieu E, Shine KP, and Clarkson RJ. Calculating and communicating ensemble-based volcanic ash dosage and concentration risk for aviation. $\mathrm{Me}$ teorological Applications, Dec 2018. DOI: 10.1002/met.1759.

[11] Giehl C, Brooker RA, Marxer H, and Nowak M. An experimental simulation of volcanic ash deposition in gas turbines and implications for jet engine safety. Chemical Geology, 461:160170, Jun 2017. DOI: 10.1016/J.CHEMGEO.2016.11.024.

[12] Bons JP, Prenter R, and Whitaker S. A Simple Physics-Based Model for Particle Rebound and Deposition in Turbomachinery. Journal of Turbomachinery, 139(8):081009-1-12, Mar 2017. DOI: $10.1115 / 1.4035921$.

[13] Filippone A. Comprehensive analysis of transport aircraft flight performance. Progress Aero. Sciences, 43(3):192-236, Apr 2007. DOI: 10.1016/j.paerosci.2007.10.005.

[14] Filippone A. Theoretical framework for the simulation of transport aircraft flight. J. Aircraft, 47(5):1669-1696, Sept 2010. DOI: 10.2514/1.C000252.

[15] Filippone A. Advanced Aircraft Flight Performance. Cambridge Univ. Press, 2012.

[16] Zhang M, Filippone A, and Bojdo N. Multi-objective optimisation of aircraft departure trajectories. Aerospace Science \& Technology, 79:37-47, Aug 2018. DOI: 10.1016/j.ast.2018.05.032.

[17] Inness A, Ades M, Agustí-Panareda A, Barré J, Benedictow A, Blechschmidt AM, Dominguez JJ, Engelen R, Eskes H, Flemming J, Huijnen V, Jones L, Kipling Z, Massart S, Parrington M., Peuch VH, Razinger M, Remy S, Schulz M, and Suttie M. The CAMS reanalysis of atmospheric composition. Atmospheric Chemistry \& Physics, 19(6):3515-3556, 2019. DOI: 10.5194/acp-19-3515-2019.

[18] Morcrette JJ, Boucher O, Jones L, Salmond D, Bechtold P, Beljaars A., Benedetti A, Bonet A, Kaiser JW, Razinger M, Schulz M, Serrar S, Simmons AJ, Sofiev M, Suttie M, Tompkins AM, 
and Untch A. Aerosol analysis and forecast in the european centre for medium-range weather forecasts integrated forecast system: Forward modeling. Journal of Geophysical Resesearch, 114(D6), June 2009. DOI: 10.1029/2008JD011235.

[19] Kern D and Seaton R. A Theoretical Analysis of Thermal Surface Fouling. Br. Chem. Eng., 4:258-262, 1959.

[20] Bojdo N, Ellis M, Filippone A, Jones M, and Pawley A. Particle-Vane Interaction Probability in Gas Turbine Engines. Journal of Turbomachinery, 141(9), Sept 2019. DOI: $10.1115 / 1.4043953$.

[21] Bojdo N and Filippone A. A Simple Model to Assess the Role of Dust Composition and Size on Deposition in Rotorcraft Engines. Aerospace, 6(4):44, apr 2019. DOI: 10.3390/aerospace6040044.

[22] Döring F, Staudacher S, Koch C, and Weißschuh M. Modeling Particle Deposition Effects in Aircraft Engine Compressors. Journal of Turbomachinery, 139:1-10, 2017. DOI: $10.1115 / 1.4035072$.

[23] Smialek JL, Archer FA, and Garlick RG. Turbine airfoil degradation in the Persian Gulf war. Journal of Minerals, 46(12):39-41, Dec 1994. DOI: 10.1007/BF03222663.

[24] Boulanger A, Patel H, Hutchinson J, DeShong W, Xu W, Ng W, and Ekkad S. Preliminary Experimental Investigation of Initial Onset of Sand Deposition in the Turbine Section of Gas Turbines. In ASME Turbo Expo 2016, Volume 1: Aircraft Engine; Fans and Blowers; Marine, page V001T01A003. ASME, Jun 2016. DOI: 10.1115/GT2016-56059. 